\title{
Demencia reversible por déficit de vitamina B12 en un adulto mayor
}

Leonidas Carrillo Ñañez ${ }^{1, a}$, Pamela Carrillo García ${ }^{2, b}$, Mirna Varas Álvarez ${ }^{3, a}$, Fernanda Aliaga Córdova ${ }^{4, a}$, César Chian García $a^{5, a}$

\section{RESUMEN}

Se presenta el caso de un paciente adulto mayor con antecedente de haber presentado úlcera gástrica hace 40 años, cuyos familiares observaron desde hace dos meses cambios en el comportamiento, los cuales incluyeron progresivamente desorientación, agitación psicomotriz, negativismo, delirio de persecución, lo que motivó ser traído al servicio de emergencia. Así mismo presentó palidez marcada y equimosis múltiple, por lo cual fue admitido posteriormente en nuestro servicio y diagnosticado de demencia reversible por anemia perniciosa. También se le detectó pancitopenia y cambios neurológicos. El paciente respondió favorablemente a la administración de vitamina B12.

Palabras clave: Demencia reversible; déficit de cobalamina; anemia perniciosa.

\section{Reversible dementia caused by vitamin B12 deficiency in an older adult}

\section{ABSTRACT}

This is the case of an elderly patient with a 40-year history of stomach ulcer, whose relatives perceived behavioral changes for the last two months. Those changes progressively included disorientation, psychomotor agitation, negativism and delirium of persecution, which caused him to be brought to the emergency room. He also showed marked pallor and multiple ecchymosis, due to which he was hospitalized in our service and diagnosed with reversible dementia caused by pernicious anemia. Pancytopenia and neurological changes were also found. The patient responded favorably to the administration of vitamin B12.

Keywords: Reversible dementia; cobalamin deficiency; pernicious anemia.

1. Médico Internista, Profesor de medicina Universidad Nacional Mayor de San Marcos y Universidad de San Martín de Porres.

2. Médico Residente IV Geriatría.

3. Médico Residente III Medicina Interna.

4. Interna de Medicina Universidad de San Martín de Porres.

5. Médico Patólogo, Profesor Universidad Particular Cayetano Heredia.

a. Hospital Nacional Arzobispo Loayza. Lima, Perú.

b. Hospital Clínica "San Carlos”. Madrid, España. 


\section{INTRODUCCIÓN}

Mejores avances en medicina en los últimos años, ha resultado en un aumento dramático en la expectativa de vida, esto sin embargo ha sido acompañado por un incremento en la prevalencia de enfermedades crónica relacionada a la edad, con morbilidad, incapacidad y pobre calidad de vida frecuentemente presente en adultos mayores. El pobre estado nutricional es reconocido como un factor contribuyente y recientemente el rol de la vitamina B12 en el envejecimiento ha atraído una considerable atención, aparte de las características clínicas de la deficiencia de B12, evidencia emergente indican que deficiencias subclínicas de la misma puede estar implicado en diversas enfermedades crónicas relacionadas con la edad ${ }^{(1)}$.

La deficiencia de vitamina B12 ha sido altamente vinculadas a diversos desordenes psiquiátricos, como pérdida de memoria, irritabilidad, depresión, demencia, delirium, esquizofrenia y psicosis ${ }^{(2)}$. Otras manifestaciones comúnmente asociadas incluyen manifestaciones neurológicas, como parestesias, entumecimiento, y degeneración subaguda combinada de la médula espinal ${ }^{(3)}$.

Este déficit vitamínico cobra relevancia al participar la cobalamina en la formación de purinas y pirimidinas, afectando la duplicación celular, así, la alteración de dichos procesos en el SNC puede causar enfermedades neurológicas o psiquiátricas, entre ellos deterioro cognitivo y demencia ${ }^{(4)}$. Diversas prevalencias de déficit de vitamina B12 han sido reportado entre paciente con diferentes condiciones psiquiátricas. Estudios publicados tienen prevalencia de $29 \%$, $44 \%$ y $70.8 \%$ entre los pacientes con demencia primaria, depresión y esquizofrenia ${ }^{(4,5)}$. Sin embargo, la prevalencia de deficiencia que se estima como responsable de demencia reversible es en promedio de $1 \%$ de todas las causas de demencia.

Los síndromes demenciales se consideran frecuentes en los adultos mayores, y dentro de la variedad de subtipos, la deficiencia de vitaminas como la B12 puede desencadenar un cuadro de deterioro cognoscitivo de patrón subcortical potencialmente reversible con el tratamiento actual, sobre todo en fases tempranas ${ }^{(6)}$. Estas manifestaciones psiquiátricas se dan en el contexto de una anemia megaloblástica, que de manera habitual se manifiesta por síntomas hematológicos, gastrointestinales y neuropsiquiátricas, por tal motivo el reconocimiento temprano de este síndrome clínico ante un paciente con manifestaciones descritas permiten tras la restitución de la deficiencia vitamínica controlar los trastornos que derivan de ella.

Presentamos el caso de un paciente adulto mayor con demencia reversible por deficiencia de vitamina B12 secundaria a anemia perniciosa asociado a vitíligo localizado focal. Al tratamiento con cobalamina el paciente evolucionó de manera favorable.

\section{CASO CLÍNICO}

Paciente de 76 años, jubilado, procedente de Lima, con instrucción secundaria completa fue admitido en nuestro servicio de medicina por haber presentado cansancio, fatiga, astenia, hiporexia, disnea a pequeños esfuerzos, dificultad para ponerse de pie. Desde hace dos meses familiares observaron moretones de manera espontánea, cambios en el comportamiento, irritabilidad, negativismo, agitación psicomotora, delirio de persecución, baja de peso $6 \mathrm{~kg}$. Antecedentes patológicos: úlcera gástrica hace 40 años, hemorroides hace 30 años, accidente de tránsito en el 2004. Sin antecedente de enfermedad mental.

Examen físico : presión arterial : 105/60 mmHg , frecuencia cardiaca : $60 \times \mathrm{m}$, frecuencia respiratoria: $22 \times \mathrm{m}$, temperatura : $36.40 \mathrm{C}$, peso $54 \mathrm{Kg}$, talla 1.54 $\mathrm{m}$. Aparente regular estado general. Aparente regular estado de nutrición. Buen estado de hidratación. Piel tibia, seca, elástica, palidez marcada, equimosis en extremidades superiores, máculas hipocrómicas en dorso y planta de pies, en región de glande. No adenopatías. Tejido celular subcutáneo: normal. Pulmones murmullo vesicular pasa bien en ambos hemitorax. Cardiovascular : ruidos cardiacos de buena intensidad, rítmicos, no soplos. Abdomen semiológicamente sin alteraciones, genitourinario: normal. Neurológico: despierto desorientado en tiempo y espacio. Funciones mentales alteración del cálculo, memoria reciente y praxia. Marcha incremento de base de sustentación Glasgow 15/15. Reflejos osteotendinosos: rotuliano $+/++$ aquiliano $\mathrm{O} /++$. Minimental State Examination (MMSE) 24/30 con un test del reloj ordenado (TRO) de 4 /10 como se puede observar en la figura 1. 
Exámenes auxiliares:

\begin{tabular}{|c|c|c|c|}
\hline Hematocrito & $14.70 \%$ & Bilirrubinas total & $2.44 \mathrm{mg} \%$ \\
\hline Hemoglobina & $5.10 \mathrm{gr} / \mathrm{dL}$ & Bilirrubina directa & $1.43 \mathrm{mg} \%$ \\
\hline Leucocitos & $3020 \times \mathrm{mmc}$ & Bilirrubina Indirecta & $1.01 \mathrm{mg} \%$ \\
\hline Abastonado & $4 \%$ & Grupo y Factor & $\mathrm{ARH}+$ \\
\hline Segmentado & $58 \%$ & VGM & $102.80 \mathrm{fL}$ \\
\hline Neutrófilo & $62 \%$ & HBGM & 37.70 \\
\hline Eosinofilos & $2 \%$ & CHBGM & $36.60 \%$ \\
\hline Monocitos & $1 \%$ & FSP & Anisocitosis $3+$ \\
\hline Macrocitosis $3+$ & $35 \%$ & $\mathrm{DHL}$ & Macrocitosis 3+ \\
\hline Linfocitos & $35 \%$ & $\mathrm{DHL}$ & $4066 \mathrm{U} / \mathrm{L}$ \\
\hline Plaquetas & $90.000 \times \mathrm{mmc}$ & AC anti células parietales & 49.3 positivo $>15$ \\
\hline (Elisa) & $20.6 \%$ & Ferritina & (Elisa) \\
\hline Hierro sérico & $44.9 \mathrm{ug} / \mathrm{dL}$ & Transferrina & $218 \mathrm{ug} / \mathrm{dL}$ \\
\hline Índice de saturación de transferrina & $20.6 \%$ & Ferritina & $964.5 \mathrm{ng} / \mathrm{ml}$ \\
\hline B12 & $55.75 \mathrm{pg} / \mathrm{ml}$ & ácido fólico & $14.70 \mathrm{ng} / \mathrm{ml}$ \\
\hline Reticulocitos & $0.4 \%$ & Hormonas tiroideas & Normal \\
\hline Glucosa & $87 \mathrm{mg} / \mathrm{dL}$ & Urea & $46 \mathrm{mg} / \mathrm{dL}$ \\
\hline Creatinina & $0.72 \mathrm{mg} / \mathrm{dL}$ & Albumina & $3.32 \mathrm{gr} / \mathrm{dL}$ \\
\hline TSH & 4.37 & $\mathrm{~T} 4 \mathrm{~L}$ & 1.06 \\
\hline HAPTOGLOBINA & $<5$ & & \\
\hline
\end{tabular}

Radiografía de tórax sin alteraciones parenquimal. Ecografía abdominal: hepatopatía difusa, esteatosis hepática leve a moderada. Litiasis vesicular múltiple. HBP II.

Se realizó endoscopia digestiva alta (Figura 3): fue informado como pangastritis atrófica, esofagitis no erosiva. Biopsia gástrica mostró (Figura 4): gastritis crónica moderada, daño mucoso moderado, metaplasia intestinal. Escasas glándulas parietales y leve infiltrado linfocítico.

Fueron los diagnósticos del servicio: Anemia perniciosa manifestada principalmente por pancitopenia y demencia megaloblástica, vitíligo, litiasis vesicular, HBP II.

Evolución: presentó episodios de agitación psicomotriz, negativismo, actitud de huida, se aplicó haloperidol, llegándose a controlar, durante su estancia en emergencia le transfundieron 1 PG por la anemia. Al séptimo día de tratamiento con B12 IM más ácido fólico se solicitó recuento de reticulocitos encontrándose $20 \%$, se evidenció mejoría clínica, una valoración por psiquiatría concluyó en el momento de la evaluación tratarse de un delirium más deterioro cognitivo.

Dos semanas después se tomó un control hematológico encontrándose $\mathrm{Hb} 9.20 \mathrm{~g} \%$ Hto $31 \%$ Leucocitos 6,380 $\mathrm{x} \mathrm{mmc}$, plaquetas 495,000 x mmc, desde el punto de vista clínico se observó un paciente tranquilo orientado en las tres esferas, colaborador se evaluó la parte neurológica presentaba aún dificultad para la marcha , lo hacía con ayuda, hiporeflexia tendinosa, se reevaluó MMSE obteniéndose 25/30 y con mejores resultados en TRO $9.5 / 10$, como se puede observar en la figura 2 . Una semana después de su alta en el control ambulatorio se observó a un paciente orientado en tiempo espacio, persona, colaborador, tranquilo sólo con indicaciones de cobalamina IM y ácido fólico por vías oral.

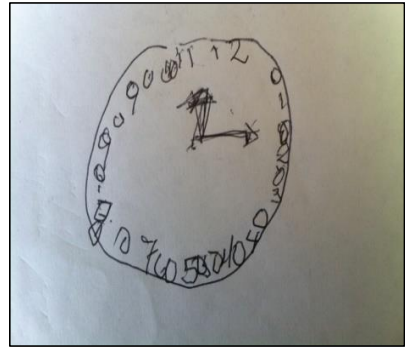

Figura 1 TRO 4/10

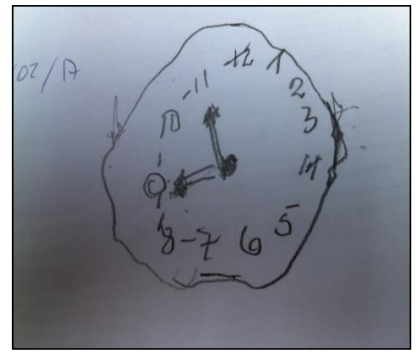

Figura 2

TRO 9.5/10

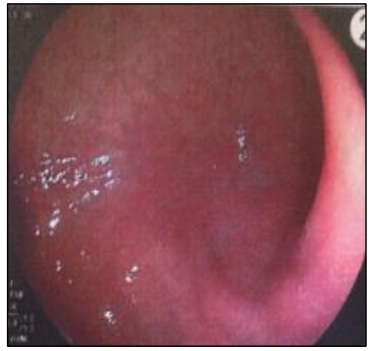

Figura 3

Pangastritis atrófica

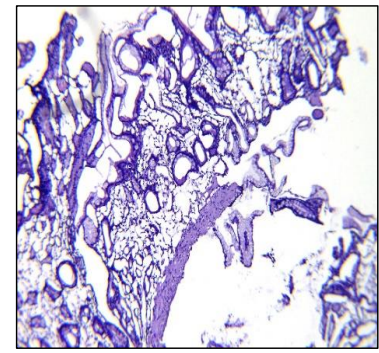

Figura 4

Escasa glándulas parietales, metaplasia 


\section{DISCUSIÓN}

El trastorno del estado de conciencia es una manifestación común en los pacientes admitidos en el servicio de emergencia de nuestro hospital, principalmente en adultos mayores, su evaluación inicial y las medidas inmediatas que se tomen harán variar la morbilidad según sea el caso. La historia clínica como el examen físico bien realizado es la herramienta principal para el acercamiento diagnóstico, así muchos trastornos de sensorio son parcialmente explicados, aunque epidemiológica y estadísticamente podrían corresponder a diagnósticos frecuentes como enfermedad cerebrovascular en alguna de sus modalidades, alteraciones metabólicas y del medio interno, sin embargo, hay otras situaciones diagnósticas que se podrían plantear inicialmente cuya característica está relacionada al tiempo y evolución de la enfermedad en discusión, como enfermedades crónicas reagudizadas, enfermedades neurodegenerativas. El caso que discutimos presenta una agrupación de síntomas y signos que revela alteraciones hematológicas y neuropsiquiátrica con indicadores laboratoriales que determinan la presencia de anemia megaloblástica del tipo de anemia perniciosa con una infrecuente forma de presentación como demencia reversible, aunque también con manifestaciones neurológicas como lo fueron dificultad para la marcha, disminución de la sensibilidad superficial y profunda, disminución marcada de los reflejos aquilianos y desde el punto de vista hematológico pancitopenia, el síndrome demencial puede ser una de las primeras manifestaciones de la deficiencia de vitamina B12. Las principales manifestaciones asociadas a la deficiencia de vitamina B12 son las alteraciones hematológicas, gastrointestinales, neurológicas, las manifestaciones psiquiátricas son poco frecuentes, siendo este el orden de observación en nuestro servicio, también han sido reportadas síntomas atípicos.

No obstante las condiciones psiquiátricas pueden ser manifestaciones solitarias o preliminares de deficiencia de vitamina B12 y pueden preceder a la manifestaciones hematológicas incluso varios meses o años ${ }^{(7)}$, la recuperación total tras la administración de cobalamina va a depender principalmente cuando esta se trate durante la etapa más temprana de la enfermedad, en caso contrario la reversibilidad total puede no ocurrir.

Es de particular interés saber que el paciente no presentaba factores de riesgo para enfermedad cerebrovascular, no ha consumido alcohol ni drogas, además de no haber registrado alteraciones de la conducta ni del comportamiento antes de enfermar, por tal razón se asume como una enfermedad de reciente comienzo y que caracteriza su potencial reversibilidad, es decir, demencia por déficit de vitamina B12, como se pudo documentar con los estudios realizados.

Los síndromes demenciales se consideran frecuentes en los adultos mayores, y dentro de la variedad de subtipos, la deficiencia de vitaminas como la B12 puede desencadenar un cuadro de deterioro cognoscitivo de patrón subcortical potencialmente reversible con el tratamiento actual, sobre todo en fases tempranas ${ }^{(6)}$. La demencia puede definirse como un síndrome clínico caracterizado por una variedad de síntomas y signos manifestados por dificultades en la memoria, trastornos en el lenguaje, cambios psicológicos y psiquiátricos, sobre todo conductuales, y deterioro en las actividades de la vida diaria ${ }^{(9)}$. Por tal razón debe el médico tratante incluir dentro de la valoración de la anemia megaloblástica la búsqueda de indicadores de alteraciones del estado mental, es decir, incluir pruebas neuropsicológicas elementales.

No está del todo clara la fisiopatología de las alteraciones neuropsiquiátricas, sin embargo, esta se explica porque la cianocobalamina participa en una serie de reacciones bioquímicas que involucran la metilación de diversos metabolitos. El daño neurológico que ocurre en su deficiencia puede ser consecuencia de las alteraciones vasculares asociadas a la elevación de la homocisteína, alteraciones en la metilmalonil CoA mutasa o a defectos en las reacciones de metilación. ${ }^{(8,9,10)}$. Estos últimos alteran la proteína básica de la mielina, lo que impide su correcta conformación o acelera su destrucción.

La causa más común de su déficit es la insuficiente ingesta en la dieta, principalmente entre vegetarianos, alcohólicos, otras causas incluye gastritis atrófica principalmente en adultos mayores, anemia perniciosa, síndromes de mala absorción, e inducido fármacos como metformina e inhibidores de bomba de protones ${ }^{(3,7,10)}$.

Sobre un estudio de 89 casos se identificaron diferentes causas de déficit de B12 y fueron agrupados en cuatro principales categorías: deficiencia nutricional en 20 casos, perdida de factor intrínseco i/o mala absorción de la proteína ligadora de B12 en 36 casos, mala absorción general 8 , competición con B12 por toxicidad con óxido nitroso, metformina 11 casos, en 14 casos no se reportó causa de deficiencia ${ }^{(10)}$.

La incidencia de síntomas cerebral en déficit de B12 es desconocido, reportes varían entre 4-50\% dependiendo de la población estudiada y la definición usada de deficiencia de vitamina B12, siendo sus efectos cambio de humor (agitación, depresión, manía), episodio psicótico (paranoia, alucinaciones auditivas y visuales, delusiones), disfunción cognitiva (11,12). Han sido descritos casos de demencia con disfunción cognitiva, demencia asociada a delirium ${ }^{(13,14)}$. Diagnosticada la anemia megaloblástica y evaluado de manera sistémica sus efectos, se debe iniciar tratamiento, para el caso particular que presentamos la obtención de buena respuesta dependió que tan temprano se inició la reposición de vitamina. Así el tratamiento tiene tres objetivos: a) corregir la anemia; b) prevenir y estabilizar las manifestaciones clínicas neurológicas y psiquiátricas c) normalizar los niveles de vitamina B12. Existen diferentes pautas $1.000 \mathrm{mcg}$ al día por vía intramuscular de cianocobalamina durante 2 semanas. A los 5-7 días se empieza a observar reticulocitosis y a las 2-3 semanas se aprecia la corrección de las alteraciones hematológicas y bioquímicas, por lo que se disminuye la dosis a 1.000 mcg por semana durante 1-2 meses, hasta que se corrija la clínica y los parámetros analíticos. En el caso de anemia perniciosa o en la gastrectomía el tratamiento es de por vida, con una dosis de cianocobalamina intramuscular al mes ${ }^{(15)}$. Cuando exista deficiencia de ácido fólico es importante descartar el déficit de vitamina B12 antes de comenzar el tratamiento con ácido fólico, ya que si solamente se trata la carencia de folato se pueden agravar las manifestaciones neuropsiquiátricas. La respuesta al tratamiento provee confirmación diagnóstica. La respuesta hematológica comienza aproximadamente 1 semana después de iniciado el tratamiento. No existe claridad sobre la respuesta a nivel neurológico ${ }^{(16,17,18)}$. 
Aunque la anemia perniciosa es generalmente raro, el diagnóstico es más frecuente en el anciano en combinación con otras enfermedades autoinmunes, como ocurrió con nuestro paciente portador de vitíligo, si el paciente no ha tenido historia de gastrectomía se tiene que investigar anticuerpos contra el factor intrínseco y contra las células parietales, concomitantemente el cáncer gástrico puede estar presente en el 10\%. El tratamiento en estos casos es por vía parenteral y sin interrupción ${ }^{(19,20)}$. La evaluación clínica de pacientes con deficiencia de vitamina B12 debe incluir una historia de consumo de fármacos, búsqueda de síndrome de mala absorción e investigación de causas autoinmunes de deficiencia de vitamina B12, tal como anemia perniciosa ${ }^{(21)}$.

En conclusión presentamos el caso de un paciente adulto mayor con un cuadro clínico de demencia por déficit de vitamina $\mathrm{B} 12$, cuya reversibilidad fue observada tras la reposición de cobalamina y ácido fólico intramuscular diariamente por una semana , luego cobalamina IM semanal y ácido fólico por vía oral, durante su hospitalización , es importante valorar cambios del estado mental por más sutiles que fuesen a fin de no catalogar al anciano como portador de una enfermedad neurodegenerativa o vascular como causa de síndrome demencial.

\section{REFERENCIAS BIBLIOGRÁFICAS}

1. Hughes C, Ward M, Hoey L, McNulty H. Vitamin B12 and ageing: current issues and interaction with folate. annals of Clinical Biochemistry. 2013; 50(4): 315-29.

2. Moore E, Mander A, Ammes D, Carne R, Sanders K, Watters D. Cognitive impairment and vitamin B12: a review. int Psichogeriatric. 2012;24(4):541-56.

3. Robert C, David L. vitamin B12 deficiency. american Family Physician. 2003; 67(5): 979-86.

4. Penninx B, Guralnik J, Ferrucci L, Fried L, Alien R, Stabler S. Vitamin B12 deficiency and depression and aging sttudy in physical disabled older women : epidemiologic evidence from the wome'ns health. Am J Psychiatry. 2000; 157(5): 715-21.

5. Silver H. Vitamin B12 levels are low in hospitalised psychiatric patient. J Psyquiatric. 2000; 37(1):41-5.

6. Goebels N, Soyka M. dementia associated with vitamin B12 deficiency : presentation of two cases and review of the literature. J Neuropsychiatry Clin Neurosci. 2000;12(3):389-95.

7. Andres E, Loukili N, Noel E, Kaltenbach G, Abdelgheni M, Perrin A, et al.Vitamin B12 (cobalamin) deficiency in elderly patients. CMAJ. 2004;171(3): 251-9.

8. Kibirige D, Wekesa C, Kaddu-Mukasa M, Waiswa M. Vitamin B12 deficiency presenting as an acute confusional state: a case report and review of literature. Afr Health Sci.2013;13(3):850-2
9. Burns A, Ilffe S. Alzheimer's disease. BMJ. 2009; 338:b158.

10. Rusher D, Pawlak R. A review of 89 published Case studies of Vitamin B12. J Hum Nutr Food Sci. 2013;1(2):1008.

11. Favrat B, Vaucher P, Herzig L, Burnand B, Ali G, Boulat O, et al. Oral vitamin B12 for patients suspected of subtle cobalamin deficiency:a multicentre pragmatic randomized controlled trial. BMC Fam Pract. 2011; 12: 2.

12. Lerner $\mathrm{V}$, Kanevsky $M$. Acute dementia with delilrium due to vitamin b12 deficiency : a case report. Int J Psychiatry Med. 2002; 32(2): 215-20.

13. De Paz R, Hernandez-Navarro F, Reinoso-Perez F, Rivas-Pollmara I. Diagnostico y tratamiento de las anemias megaloblasticas. Medicine. 2008; 10(20):1326-33.

14. Carmel R. How I treat cobalamin (vitamin B12) deficiency. Blood. 2008; 112(6):2214-21.

15. Schrier S. Etiology and clinical manifestations of vitamin B12 and folic acid deficiency. En UpToDate, Mentzer W (ed), UpToDate, Waltham M, 2011.

16. Erkurt M, Aydogdu I, Dikilitas M, kuku I, Kaya E, Bayraktar N, et al. Effect of cyanocobalamin on inmunity in patient with pernicious anemia. Med Princ Pract. 2008; 17(2): 131-5.

17. OHTA M. Management of anemia in the elderly. JMAJ. 2009; 52(4): 219-23.

18. Emmanuel A, Khalid S. Optimal management of pernicious anemia. J Blood Med. 2012; 3: 97-103.

19. Andre E, Loukili N, Noel E, Kaltenbach G, Abdelgheni M, Perrin A, et al.Vitamin B12 deficiency in Elderly Patients. CMAT. 2004; 171(3): 251-9.

Fuentes de financiamiento:

Este artículo ha sido financiado por el autor.

Conflictos de interés:

El autor declara no tener ningún conflicto de interés.

\section{Correspondencia:}

Leonidas Carrillo Ñañez

Dirección: Calle los Ficus 136 Urbanización Jardines Viru -

Bellavista

Teléfono: 945330799

Correo electrónico: Ichcarn@yahoo.es

Recibido: 27 de febrero de 2017 Aprobado: 03 de mayo de 2017 